\title{
Effect of a fetal surveillance unit on admission of antenatal patients to hospital
}

\author{
P W Soothill, R Ajayi, S Campbell, J Gibbs, R Chandran, D Gibb, K H Nicolaides
}

\begin{abstract}
Objective - To analyse the effect of a fetal surveillance unit, which undertakes a wide range of maternal and fetal tests on an outpatient or inpatient basis, on the number and length of antenatal hospital admissions.
\end{abstract}

Design-A comparison of the number and length of antenatal admissions six months before and five months after the opening of the unit on 1 July 1990.

Main outcome measures-Admission rate, antenatal bed occupancy, and interval from admission to discharge or delivery.

Results - The antenatal bed occupancy rate fell by $22 \%$ from $174 / 100$ deliveries during the six months before the unit was opened to $136 / 100$ deliveries in the five months after it was opened. The difference in distribution of lengths of admission after the unit was opened from before was highly significant (Mann-Whitney test $=5 \cdot 14, \quad n=752$ and 679 ; $\mathbf{p}<0.0001$, and this was due to shorter intervals from admission to discharge and from admission to delivery. In contrast, the antenatal admission rate did not change significantly $(50 / 100$ deliveries $v 49 / 100$ deliveries). There was no significant change in the stillbirth rate $(6 / 1294$ births $v 8 / 1372$ births; difference between rates $=0.0012,95 \%$ confidence interval -0.0043 to 0.0067 ).

Conclusion-Obstetricians are more prepared to discharge antenatal patients from hospital and, similarly, admit patients for delivery rather than for assessment if the patients are reliably monitored on an outpatient basis. If this change in practice is sustained substantial financial and social benefits will result as well as improvements in organisation, audit, teaching, and research.

\section{Introduction}

Pregnant women are admitted to hospital for one of three reasons: to receive treatment that cannot be obtained at home; because they are at risk of an emergency complication that requires rapid intervention; or for initial assessment and subsequent serial monitoring of maternal or fetal disease. The investigation of fetal wellbeing entails performing tests that can nearly always be done within 90 minutes, and for many clinical indications if the results are normal the tests will not need to be repeated. With other complications in pregnancy serial investigation is required (for example, every week), but only very rarely is it necessary to repeat the tests more than once a day. Therefore, assessment of fetal wellbeing can nearly always be done on an outpatient basis provided the patient is mobile and lives within a reasonable distance from the hospital. Indeed, admission to hospital is expensive and may have serious social consequences for the patient.

We set up a unit that can undertake maternal and fetal assessment on an outpatient basis. In this study we examine the effect of the unit on hospital admissions and bed occupancy.

\section{Methods}

\section{FETAL SURVEILLANCE UNIT}

The fetal surveillance unit was established within the maternity department of King's College Hospital. It has six beds in four rooms, is staffed by a midwifery sister and two health care assistants, and is supervised by PWS. The first patient was seen on 4 June 1990, but the first few weeks were used to learn techniques, establish procedures, and inform the obstetricians in the hospital about the facilities. The unit opened fully on 1 July 1990. Any registrar or consultant at the hospital can refer a patient with a viable fetus $(>26$ weeks' gestation), but the patient remains under the care of the referring consultant and the staff of the unit do not make any management decisions. The indication for referral and the action that would have been taken if the unit had not been available is stated on the request form. The referring doctor also states a plan for follow up if the test results are normal (that is, review in the antenatal clinic or weekly assessment in the unit). The patient is reviewed by the referring obstetric team in the unit if any of the test results are abnormal.

On referral the demographic details are taken, as is a brief obstetric history and review of the obstetric notes, which in our hospital are carried by the patients. Every visit includes taking a further brief history, monitoring maternal variables such as blood pressure, urine testing, measurement of symphysis-fundal height, and, if requested by the referring doctor, maternal blood testing for conditions which could affect the fetus, such as anaemia, thrombocytopenia, and hyperglycaemia. The following fetal tests are done at every visit by a midwifery sister: assessment of the fetal heart rate by cardiotocography ${ }^{1}$ (usually with computer analysis ${ }^{2}$ ), ultrasound studies of fetal behaviour expressed as the biophysical profile ${ }^{3}$ and Doppler studies of the fetoplacental ${ }^{4}$ and uteroplacental ${ }^{5}$ circulation. The results are entered into a specially written computer program which produces a clinical spread sheet, which is inserted into the notes carried by the patient.

\section{MEASUREMENT OF UNIT'S EFFECT}

Antenatal admission figures were obtained from the midwifery ward diaries and the figures for the six months before the unit was opened were compared with those for the five months after. The figures are expressed in three ways:

Antenatal admission rate-This is the incidence of antenatal admissions. If a woman was admitted to the labour ward, delivered her baby, and then was trans-
K H Nicolaides, MRCOG, consultant

$B M \mathcal{F}$ 1991;303:269-71 
ferred to the other wards as a postnatal patient no antenatal admissions were recorded. However, if she was transferred before delivery from the labour ward or admitted directly to an antenatal ward whether she stayed for a few hours or many weeks this was recorded as one antenatal admission. The rate is expressed per 100 deliveries to allow for variations in the number of women delivering at the hospital.

Bed occupancy-This is the summation of the number of beds occupied by all antenatal patients over the study period (expressed in bed days). Thus an antenatal patient admitted for seven days would have the same bed occupancy as seven women admitted for one day, although there would be a sevenfold difference in the number of antenatal admissions. Bed occupancy is also expressed per 100 deliveries.

Intervals from admission to discharge and from admission to delivery-For statistical analysis the intervals from admission to discharge and from admission to delivery were counted in days for each patient. When these admissions crossed our study limits (for example, started before but continued after the unit opened or started in but continued after the end of the study period) the full length of the admission was included as if the whole admission was in the month of admission. This analysis could have biased the data, but as one patient's admission continued for 54 days after the study period any bias reduced the significance of the unit's affect on admissions. As the admission interval was not normally distributed even after logarithmic transformation the data were compared by the nonparametric Mann-Whitney test by using the Statistical Package for Personal Computers (Timberlake Clarke). ${ }^{6}$

The number of deliveries per month and the number of stillbirths (babies born without signs of life at or after 26 weeks of gestation) were obtained from the labour ward's computer and compared by using the $\chi^{2}$ test with Yates's correction. The gestational age limit of 26 weeks was chosen because this is the minimum gestational age at which pregnancies can be referred to the unit.

\section{Results}

From 1 July 1990 to 30 November 1990,330 patients were referred to the unit and were seen on 754 occasions. The table gives indications for referral specified by the referring doctor. At initial referral the doctors stated on 85 occasions that admission to hospital would have been arranged if the unit had not been available.

The figure shows the effect of the unit on antenatal admissions. The rate of admission did not fall significantly after the unit was opened compared with during the previous six months (50 $v 49$ antenatal admissions per 100 deliveries). There was, however, a $22 \%$ decrease in the antenatal bed occupancy per 100 deliveries (136 $v 174$ beds occupied). The distribution of length of admission was significantly different after the opening of the unit (Mann-Whitney $=5 \cdot 14, n=752$

Indications for referral to fetal surveillance unit as stated by referring obstetrician

\begin{tabular}{lc}
\hline Indication & $\begin{array}{c}\text { No }(\%) \\
\text { of referrals }\end{array}$ \\
\hline Decreased fetal movements & $59(18)$ \\
Pregnancy induced hypertension & $43(13)$ \\
Post-term pregnancy & $40(12)$ \\
Antepartum haemorrhage & $40(12)$ \\
Small for gestational age & $33(10)$ \\
Other reasons & $30(9)$ \\
Multiple pregnancies & $30(9)$ \\
Spontaneous rupture of membranes & $20(6)$ \\
Abdominal pain & $17(5)$ \\
Poor obstetric history & $10(3)$ \\
Abnormal uterine artery on Doppler screening & $7(2)$ \\
Rhesus isoimmunisation & $1(>1)$ \\
\hline
\end{tabular}

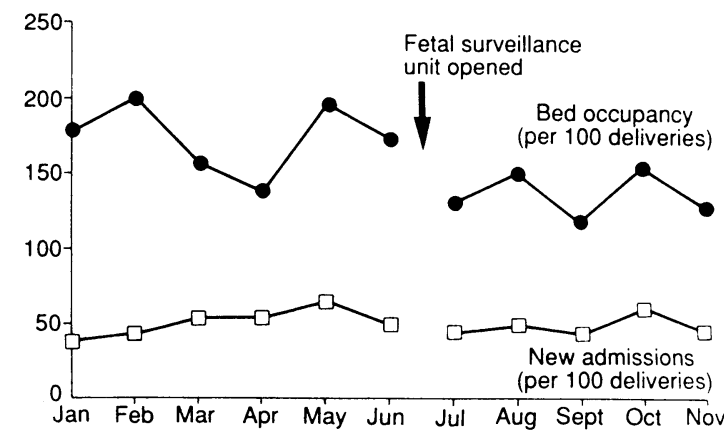

Antenatal admission rate and bed occupancy for six months before and five months after fetal assessment unit was opened on 1 fuly 1990

and $679, \mathrm{p}<0.0001$ ) and $70 \%$ of the reduction in mean length of admission was because of a shorter interval from admission to delivery and $30 \%$ because of a shorter interval from admission to antenatal discharge.

There were 14 stillbirths in 2666 deliveries, giving a rate of stillbirth of 5.3 per 1000 . There was no significant increase after the opening of the unit $(6$ per 1294 deliveries before and 8 per 1372 deliveries after; $\chi^{2}$ test with Yates's correction $=0.42, p=0.336$; difference between rates $=0.0012,95 \%$ confidence interval -0.0043 to 0.0067 ).

\section{Discussion}

Admission to hospital is expensive and often causes a major disruption to the woman's life. It is extremely inefficient to admit patients simply to ensure that the appropriate investigations are organised and checked. Assessment of fetal condition can almost always be done on an outpatient basis and admission can be reserved until the decision to deliver has been made. This concept is completely separate from the controversial issue as to whether bed rest is of therapeutic value in various obstetric complications. ${ }^{7}$

Another important advantage of assessing fetal wellbeing in one unit is that data can be collected for research. The tests that are used were introduced after little monitoring of their effect or controlled evidence of their value. ${ }^{8}$ By having all the tests organised into one database comparison of the tests and investigation of current practice becomes much easier. Such an approach will also make intervention studies in controversial subjects more possible in the context of a hospital with several obstetric consultants. Similarly a concentration of experience makes teaching more reliable, and people could learn the techniques of fetal wellbeing efficiently in such a unit.

The absence of any fall in the rate of antenatal admission is not surprising because whenever the decision to deliver electively a high risk pregnancy has been made the patient is admitted before induction or caesarean section. Therefore, the number of admissions would be the same as if she had been admitted only for a day or had been in hospital for weeks before delivery. Furthermore, many patients present to the labour ward with symptoms such as very early labour, antepartum haemorrhage, or possible ruptured membranes and require a short period of observation, which the presence of a fetal surveillance unit would not affect.

The fall in antenatal bed occupancy of about $20 \%$ is remarkable. The shorter interval from admission to delivery was the biggest component of this change, which suggests that after the unit had opened patients were more often admitted after the decision to deliver had been made. There was also a shorter interval from admission to antenatal discharge, indicating that the obstetricians had greater confidence to allow patients to go home when reliable follow up was ensured. 
This study compared our admission policy before and after an intervention, and it is difficult to exclude the possibility that a different but coincident change could have affected the results, especially as we were unable to have a separate control grouj. However, there was no evidence of a changing admission policy during the six months before the unit opened. The only change in our practice during the study period was the moving of obstetric beds from Dulwich Hospital to King's College Hospital. This occurred on 14 September and the reduction in bed occupancy was seen before that date. By expressing the results per 100 deliveries we allowed for the increase in the number of deliveries that happened at this time and there was no evidence of a change in admission policy after this reorganisation.

From the health service's point of view the savings to be gained from a fetal surveillance unit are clear. The cost of a hospital bed is difficult to quantify, but $£ 300$ a day is a conservative estimate. Our results suggest that in our hospital about 38 antenatal bed days can be saved for every 100 deliveries. A unit with 4000 deliveries a year could therefore save $38 \times 40=1520$ bed days, which is equivalent to $£ 456000$ a year. Although the cost of the unit must be subtracted from this figure, in view of the better patient care and saving of our patients' time, this is a cost effective reorganisation.

We thank Miss F Reid, Department of Public Health, King's College Hospital, for statistical help and the obstetric consultants of King's College Hospital for cooperation with the unit. Dr C Redman, John Radcliffe Maternity Hospital, Oxford, and Dr J Walker, Royal Infirmary, Glasgow, gave valuable advice. RA was supported by a grant from the Locally Organised Research Scheme, South East Thames Health Authority, and RC by a grant from the Commonwealth Scholarship Commission, United Kingdom.

1 Druzin ML. Antepartum fetal heart rate monitoring. State of the art. Clin Perinatol 1989;16:627-42.

2 Dawes GS, Houghton CRS, Redman CWG, Visser G. Pattern of the normal human fetal heart rate. Br f Obstet Gynaecol 1982;89:276-84.

3 Manning FA. The fetal biophysical profile score: current status. Obstet Gynecol Clin North Am 1990;17:147-62.

4 Trudinger B. The umbilical circulation. Seminars in Perinatology 1988;11: $311-21$

5 Campbell S, Griffin DR, Pearce JM, Diaz-Recasens JD, Cohen-Overbeek T, Wilson $\mathrm{K}$, et al. New Doppler technique for assessing utero-placental blood flow. Lancet 1983; ; $675-7$.

6 Meddis R. Unified analysis of variance by ranks. Br 7 Math Stat Psychol 1980;33:84-8.

Crowther CA Neilson JP, Verkuyl DAA, Bannerman C, Askhurst HM Br Obstet Gynaecol 1989;96:850-3.

8 De-Bono M, Fawdry RD, Lilford RJ. Size of trials for evaluation of antenatal tests of fetal wellbeing in high risk pregnancy. $\mathcal{F}$ Perinat Med 1990;18:77-87.

(Accepted 4 June 1991)

\section{Hindu Mandal Hospital, Dar-es-Salaam, Tanzania)
K L Ramaiya, MMED, specialist physician}

Department of Medicine, University of Dar-es-Salaam, Muhimbili Medical Centre, Dar-es-Salaam, Tanzania A B M Swai, MMED, senior lecturer

D G McLarty, FRCP, professor

Medical School, University of Newcastle upon Tyne, Newcastle upon Tyne NE2 4HH

R S Bhopal, MFPHM, professor, division of epidemiology and public health K G M M Alberti, FRCP, professor, department of medicine

Correspondence to: Professor Alberti.

\title{
$(2)$ \\ Prevalences of diabetes and cardiovascular disease risk factors in Hindu Indian subcommunities in Tanzania //
}

\author{
K LRamaiya, A B M/Swai, D G/McLarty, R S(Bhopal, K G M M/Alberti
}

\section{Abstract}

Objectives-To seek differences in the prevalence of diabetes mellitus and other coronary heart disease risk factors, and to identify factors associated with these differences within a Hindu Indian community.

Design-Population based cross sectional survey. Setting-Dar-es-Salaam, Tanzania.

Subjects-Of 20 Hindu subcommunities categorised by caste in Dar-es-Salaam, seven were randomly selected. $1147(76.7 \%)$ of 1495 subjects aged 15 or over participated.

Main outcome measures-Blood glucose concentrations (fasting and two hours after oral glucose loading), serum total cholesterol and serum triglyceride concentrations, blood pressure, and height and weight.

Results-The subcommunities differed substantially in socioeconomic characteristics and lifestyle. Overall, $9.8 \%$ of subjects $(109 / 1113)$ had diabetes, $17.0 \%(189 / 1113)$ impaired glucose tolerance, $14.5 \%$ (166/1143) hypertension, and $13.3 \%$ (151/1138) were obese. The mean fasting blood glucose concentration was $4.9 \mathrm{mmol} / 1$, the blood glucose concentration two hours after oral loading $(75 \mathrm{~g}) 6.0 \mathrm{mmol} / \mathrm{l}$, the total cholesterol concentration $4.9 \mathrm{mmol} / \mathrm{l}$, the serum triglyceride concentration $1.4 \mathrm{mmol} / \mathrm{l}$, and body mass index (weight $/$ height $^{2} ; \mathrm{kg} / \mathrm{m}^{2}$ ) $24 \cdot 3$ Systolic and diastolic blood pressures were 121 and $77 \mathrm{~mm} \mathrm{Hg}$ respectively. There were important intercommunity differences even after standardisation for age, sex, and body mass index - for example, in mean fasting blood glucose concentration (range 4.5 (Jains) to $5.9 \mathrm{mmol} / \mathbf{l}$ (Patels)), serum total cholestero concentration (range 4.5 (Jains) to $6.2 \mathrm{mmol} / \mathrm{l}$ (Suthars)), systolic blood pressure (range 110 (Limbachias) to $127 \mathrm{~mm} \mathrm{Hg}$ (Bhatias)), and prevalences of diabetes (range $3.4 \%$ (3/87 Limbachias) to $18 \%$ (20/111 Navnats)) and hypertension (range 5.7\%
(5/87 Limbachias) to $19.4 \%$ (43/222 Bhatias). Variables which showed significant linear correlation with subcommunity variations were entered into a multiple regression model. Intercommunity variations persisted. The Limbachia and Jain communities had the lowest prevalence of and mean values for coronary heart disease risk factors and the Bhatia and Patel communities had the highest.

Conclusions - In this series intercommunity variations in disease and risk factors might have been related to genetic, dietary, socioeconomic, and lifestyle differences but could not be explained by the characteristics studied. Studies of Indian subcommunities are warranted to confirm and extend these descriptive findings and explore the genetic basis of diabetes. Communities of Indian origin should not be perceived as homogeneous.

\section{Introduction}

Migrants from the Indian subcontinent in Trinidad, South Africa, ${ }^{2} \mathrm{Fiji}^{3}{ }^{3}$ Singapore, ${ }^{45}$ United Kingdom, ${ }^{6}$ Mauritius, ${ }^{8}$ and east Africa ${ }^{9}$ have a higher prevalence of diabetes or an increased frequency of coronary heart disease ${ }^{10-18}$ compared with other ethnic groups residing in the same countries. Several ethnic groups from the Indian subcontinent (Gujarati, Punjabi, Bangladeshi, and Pakistani) have been shown to have a $50 \%$ higher mortality from coronary heart disease than the national average in the United Kingdom. ${ }^{19}$ The high rates of coronary heart disease cannot be explained by classic risk factors for the disease (smoking, hypertension hypercholesterolaemia) but have recently been attributed to insulin resistance and hyperinsulinaemia..$^{2021}$

Often migrants from the Indian subcontinent have been considered erroneously as a homogeneous group (so called "Asians"), but they differ in their religious, cultural, and geographical backgrounds. ${ }^{22}$ Religion, 